\title{
Estilos de Aprendizagem em Situações de Uso de Tecnologias
}

\author{
Rodrigo Hipólito Roza* \\ Pontifícia Universidade Católica de Campinas - PUC-Campinas, Campinas, SP, Brasil \\ ORCID: http://orcid.org/0000-0002-7162-4908
}

\begin{abstract}
RESUMO
Esta comunicação refere-se à tese intitulada Estilos de Aprendizagem e o Uso das Tecnologias da Informação e Comunicação, defendida no Programa de Pós-Graduação em Psicologia da Pontifícia Universidade Católica de Campinas. De modo sucinto, apresenta a sequência de quatro estudos realizados no âmbito da referida tese, sendo eles: (1) construção de um instrumento de avaliação (escala) e busca por evidências de validade baseadas em conteúdo; (2) estudo piloto para informatização da escala e teste de funcionamento da escala informatizada; (3) busca por evidências de validade baseadas na estrutura interna; e (4) comparação dos estilos de aprendizagem de universitários de administração, considerando as variáveis linhas de formação e gênero. Os estudos resultaram no desenvolvimento de um instrumento de avaliação e na proposição de um modelo de estilos de aprendizagem em situações de uso de tecnologias, abrangendo os estilos teórico, pragmático, de interação com o meio, bem como de interação através do meio.
\end{abstract}

Palavras-chave: aprendizagem, estilos de aprendizagem, tecnologia da informação e comunicação, avaliação psicológica.

\section{Learning Styles in Technology Use Situations}

\begin{abstract}
This paper refers to the thesis entitled Learning Styles and the Use of Information and Communication Technologies, defended in the Doctoral Program in Psychology of the Pontifical Catholic University of Campinas. Briefly, it presents the sequence of four studies developed in the thesis: (1) construction of an assessment instrument (scale) and search for evidence of validity based on content; (2) pilot study for scale computerization and computerized scale function test; (3) search for evidence of validity based on internal structure; and (4) comparison of the learning styles of business university students. The studies resulted in the development of an evaluation instrument and the proposition of a learning style model in technology use situations, composed by the theoretical, pragmatic, interaction with the medium and interaction through the medium styles.
\end{abstract}

Keywords: learning, learning styles, information and communication technology, psychological assessment.

ISSN $1808-4281$ 


\section{Estilos de Aprendizaje en Situaciones de Uso de Tecnologías}

\section{RESUMEN}

Este artículo se refiere a la tesis titulada Estilos de Aprendizaje y el Uso de las Tecnologías de la Información y Comunicación, defendida en el Programa de Posgrado en Psicología de la Pontificia Universidad Católica de Campinas. Brevemente, la secuencia de cuatro estudios realizados en el ámbito de dicha tesis: (1) construcción de un instrumento de evaluación (escala) y búsqueda de evidencia de validez basada en el contenido; (2) estudio piloto para la informatización a escala y prueba de función de escala computarizada; (3) búsqueda de evidencia de validez basada en estructura interna; y (4) comparación de los estilos de aprendizaje de los estudiantes universitarios de administración. Los estudios dieron como resultado el desarrollo de un instrumento de evaluación y la proposición de un modelo de estilo de aprendizaje en situaciones de uso de la tecnología, abarcando los estilos teórico, pragmático, de interacción con el medio y de interacción a través del medio.

Palabras clave: aprendizaje, estilos de aprendizaje, tecnología de la información y comunicación, evaluación psicológica.

A presente comunicação refere-se à tese Estilos de Aprendizagem e o Uso das Tecnologias da Informação e Comunicação (Roza, 2017). O objetivo principal da tese foi investigar os estilos de aprendizagem em situações de uso das Tecnologias da Informação e Comunicação (TICs), em especial de estudantes universitários de administração, tendo em vista a adequação do emprego dos recursos tecnológicos às preferências do indivíduo ao aprender. Para tanto, foram realizados quatro estudos, descritos sucintamente a seguir.

O primeiro estudo envolveu a construção de um instrumento e a busca por evidência de validade baseadas em seu conteúdo (Roza, Wechsler, \& Nakano, 2018). O instrumento construído foi chamado de Escala de Estilos de Aprendizagem em Situações de Uso de Tecnologias. Seu objetivo foi avaliar os estilos de aprendizagem dos indivíduos. Ele foi composto por 40 itens, baseado principalmente nos estudos de Alonso, Gallego e Honey (1997), cujas influências residem nas pesquisas de Kolb (1984) e Mumford e Honey (1992). Assim, incialmente foram considerados os estilos de aprendizagem ativo, reflexivo, teórico e pragmático.

O conteúdo do instrumento foi validado por quatro juízes independentes, sendo dois doutores com pós-doutorado em andamento e dois doutorandos. O processo de busca por evidências baseadas no conteúdo mostrou a adequação do instrumento aos estilos que se propunha a avaliar. 
Em seguida, foi realizado um estudo piloto para informatização do instrumento construído e teste do instrumento informatizado (Roza, Nakano, Silva, \& Wechsler, 2018). Neste segundo estudo, a informatização do instrumento abrangeu as seguintes atividades: análise e seleção de ferramentas de software preferencialmente gratuitas; testes isolados e integrados das ferramentas selecionadas; implementação informatizada da escala; e aplicação da escala em laboratórios de informática.

Após análises e testes, optou-se por realizar a informatização do instrumento com ferramentas gratuitas do Google. Todas as etapas de aplicação do instrumento, incluindo acesso ao site, coleta e tratamento de dados, bem como envio de feedback ao respondente, foram automatizadas. O processo de resposta ao instrumento foi dividido em quatro fases, contemplando: Termo de Consentimento Livre e Esclarecido, conforme a Resolução 466/12 do Conselho Nacional de Saúde (CNS), dados sócio-acadêmicos, instruções gerais e o instrumento propriamente dito.

Para avaliação da aplicação da escala em laboratórios de informática, foram considerados os seguintes parâmetros: acesso ao site, processo de resposta, registro das respostas, tratamento dos dados e feedback ao participante. Participaram desta etapa 120 estudantes universitários. Os resultados obtidos também foram considerados satisfatórios.

O terceiro estudo, por sua vez, envolveu a busca por evidências de validade baseadas na estrutura interna do instrumento (Roza, Nakano, Wechsler, \& Primi, 2018). Os participantes foram 378 estudantes universitários. Neste caso, foram realizadas duas análises principais: análise fatorial exploratória (Brown, 2006; Hair, Anderson, Tatham, \& Black, 2005) e análise de cluster (Hair et al., 2005). Os resultados empíricos indicaram uma estrutura diferente da considerada inicialmente por ocasião da construção do instrumento, realizada a partir dos estudos de Alonso et al. (1997).

A análise dos resultados empíricos, à luz do referencial teórico da pesquisa, levou à proposição de um novo modelo teórico, que foi denominado Modelo de Estilos de Aprendizagem em Situações de Uso de Tecnologias. Esse novo modelo foi composto por quatro estilos de aprendizagem:

Teórico - preferência por aprender através de modelos, teorias e conceitos, bem como por meio de reflexão, usando as TICs no apoio a atividades analíticas e abstratas.

Pragmático - preferência por aprender através de atividades práticas, usando as TICs em situações reais e na resolução de problemas concretos. 
Interação Com o Meio - preferência por aprender usando as TICs para explorar coisas novas, de forma individual.

Interação Através do Meio - preferência por aprender usando as TICs para comunicação interpessoal e realização de trabalhos colaborativos, estabelecendo relações sociais.

Além disso, como parte do modelo os estilos de aprendizagem foram divididos em dois grupos conceituais. O primeiro, formado pelos estilos teórico e pragmático. O segundo, composto pelos estilos de interação - com o meio tecnológico e através do meio tecnológico. Conceitualmente, considerou-se também o cruzamento dos estilos de aprendizagem pertencentes a grupos distintos, estabelecendo quatro perfis principais, cujas designações simplificadas são: teórico com o meio, teórico através do meio, pragmático com o meio e pragmático através do meio.

No quarto estudo, a Escala de Estilos de Aprendizagem em Situações de Uso de Tecnologias foi aplicada para comparação dos estilos de aprendizagem de 322 estudantes universitários de administração. As análises foram conduzidas a partir de estatística descritiva, bem como análise multivariada da variância (Manova), e consideraram as variáveis linhas de formação e gênero. Não forram encontradas diferenças significativas de estilos de aprendizagem em relação a essas variáveis, com exceção da interação entre elas no estilo teórico.

De modo geral, verifica-se que os resultados relativos à Escala de Estilos de Aprendizagem em Situações de Uso de Tecnologias foram satisfatórios. Observa-se também que o Modelo de Estilos de Aprendizagem em Situações de Uso de Tecnologias proposto não estava previsto a priori, sendo um desdobramento dos resultados obtidos empiricamente. Por fim, é importante destacar que tanto a escala como o modelo trazem relevantes contribuições para investigações sobre estilos de aprendizagem no contexto em que as TICs são empregadas, contrapondo aspectos relativamente simples de serem operacionalizados, como teoria e prática, bem como o uso individual e social dos recursos tecnológicos. 


\section{Referências}

Alonso, C. M., Gallego, D. J., \& Honey, P. (1997). Los estilos de aprendizaje: Procedimientos de diagnostico y mejora (7a. ed.). Bilbao: Ediciones Mensajero.

Brown, T. A. (2006). Confirmatory factor analysis for applied research. New York: The Guilford Press.

Hair, J. F., Anderson, R. E., Tatham, R. L., \& Black, W. C. (2005). Análise multivariada de dados (A. S. Sant'Anna \& A. C. Neto, Trad.). Porto Alegre: Bookman.

Kolb, D. A. (1984). Experiential learning: Experience as the source of learning and development. New Jersey: Prentice-Hall.

Mumford, A., \& Honey, P. (1992). Questions and answers on learning styles questionnaire. Industrial and Commercial Training, 24(7), 10-13. doi: 10.1108/00197859210015426

Roza, R. H. (2017). Estilos de aprendizagem e o uso das tecnologias da informação e comunicação (Tese de Doutorado). Pontifícia Universidade Católica de Campinas, Campinas, SP, Brasil. Recuperado de http://tede.bibliotecadigital.puccampinas.edu.br:8080/jspui/bitstream/tede/972/2/RODRIGO\%20HIP\%C3\%93LITO\% 20ROZA.pdf

Roza, R. H., Nakano, T. C., Silva, T. F., \& Wechsler, S. M. (2018). Tecnologia no Contexto Educacional: Teste Informatizado para Avaliação de Estilos de Aprendizagem. Psicologia da Educação, (46), 11-20. doi: 10.5935/2175-3520.20180002

Roza, R. H., Nakano, T. C., Wechsler, S. M., \& Primi, R. (2018). Escala de Estilos de Aprendizagem em Situações de Uso de Tecnologias: estrutura interna. Avaliação Psicológica, 17(2), 223-232. doi: 10.15689/ap.2018.1702.14184.08

Roza, R. H., Wechsler, S. M., \& Nakano, T. C. (2018). Escala de estilos de aprendizagem em situações de uso de tecnologias: Busca por evidências de validade de conteúdo. Estudos Interdisciplinares em Psicologia, 9(1), 45-64. doi: 10.5433/22366407.2018v9n1p45

\section{Endereço para correspondência}

\section{Rodrigo Hipólito Roza}

Centro de Economia e Administração (CEA)

Rodovia Dom Pedro I, Km 136 - Parque das Universidades, Campinas - SP, Brasil. CEP 13086-900

Endereço eletrônico: rodrigo.roza@gmail.com 
Recebido em: 20/08/2019

Aceito em: 03/08/2020

\section{Notas}

* Doutor em Psicologia e Mestre em Ciência da Informação pela PUC-Campinas. Pós-doutorado pela Universidade São Francisco. Professor da PUC-Campinas.

Financiamento: o doutorado em questão foi realizado com bolsa de estudo da própria universidade (Pontifícia Universidade Católica de Campinas).

Este artigo de revista Estudos e Pesquisas em Psicologia é licenciado sob uma Licença Creative Commons Atribuição-Não Comercial 3.0 Não Adaptada. 